\author{
Gdańsk 2018, Nr. 39 \\ https://doi.org/10.26881/sgg.2018.39.06 \\ Michail L. Kotin \\ Universität Zielona Góra, Humanistische Fakultät
}

\title{
Valenz und Afinitheit ${ }^{1}$
}

\begin{abstract}
Auf den ersten Blick scheinen beide Titelbegriffe kontradiktorisch zu sein, setzt ja die klassische verbozentrische Valenztheorie, deren Anhänger auch der Geehrte ist, eine weit verstandene „Verbalität“ als konstitutives inhaltlich-propositionales Satzkriterium voraus, wobei die Finitheit von den meisten deutschen Grammatikforschern zum unveräußerlichen formalen Satzkriterium erklärt wird. So gelten z.B. sämtliche infinite Prädikationen schlechtweg als kommunikative Minimaläußerungen, doch wird ihnen der „normale“ Satzstatus abgesprochen (vgl. Zifonun et al. 1997). Auf der anderen Seite gibt es in vielen Sprachen (so in der Slavia, aber auch in älteren germanischen Sprachen, im Griechischen, zum Teil im Latein) viele vollwertige Satzstrukturen, die formal infinit sind. Auch in der deutschen Gegenwartssprache sind mehrfach satzartige Konstruktionen mit vollprädikativem Inhaltswert, dabei aber ohne finites Verb vorhanden. Viele davon fallen unter den Begriff der Afinitheit (vgl. u.a. Riecke 2012, Kotin 2014), vgl. Was vergangen, kehrt in der gleichen Gestalt nie wieder. Wird bei der Deskription der Valenzstruktur derartiger Sätze oder satzartiger Äußerungen das klassische (Engel'sche) Valenzmodell angewandt, so ergibt dies keine rasanten Unterschiede bei finiten und afiniten Satzsyntagmen, da finite Auxiliare und Kopulae darin grundsätzlich als nicht valenzfähig behandelt werden. Schwieriger wird es allerdings, wenn angenommen wird, dass Kopulae und Auxiliare zumindest eine strukturelle Valenz besitzen und Regentia bezüglich der hierarchisch niedrigeren Vollverben darstellen (vgl. Eroms 2000). In diesem Fall sollen afinite Sätze als Ellipsen eingeordnet und entsprechend syntaktisch behandelt werden. Dieses recht komplizierte und komplexe theoretische Problem der Dependenzgrammatik wird am Beispiel verschiedener Sprachen und unterschiedlicher Entwicklungsstufen, also aus synchroner, kontrastiv-typologischer und diachroner Sicht betrachtet.
\end{abstract}

Schlüsselwörter: Dependenz, Valenz, Satz, Proposition, Finitheit, Afinitheit, Verbozentrik.

Valency and non-finiteness. - Both terms seem to be contradictory to each other, since the classical verb-centric valence theory, the follower of which is also Ulrich Engel, considers the verb, and, thus, also the formal feature of its finiteness as an inalienable criterion of a sentence proposition. Thus, non-finite predications are simply considered as minimal utterances, whereas their "normal" status of clauses is mostly denied (cf. Zifonun et al. 1997). On the other hand, many languages ( Slavic languages, among others,but also old Germanic languages, Greek and partly Latin) have many full-value clause structures being formally non-finite. Similarly, modern German conveys numerous sentence-like constructions with a full-predicative propositional value. Many of them belong to the phenomenon of non-finiteness (cf., among others, Riecke 2012, Kotin 2014), as in: Was vergangen, kehrt in der gleichen Gestalt nie wieder. If in the description of the valency structure of such sentences / utterances the classical Engelian valence model is used,

1 Der Autor bedankt sich bei zwei anonymen Gutachtern für Kritik und anregende Hinweise. In der vorliegenden Endversion wird darauf nicht explizit eingegangen, aber der Text enthält „implizite“ Antworten des Verfassers auf einzelne kritische Anmerkungen zum Beitragsinhalt. 
no significant differences between finite and non-finite clauses can be registered, since within this model finite auxiliaries and copulas are considered as not valence-dependent. However, the problem becomes more complicated, if we assume that copulas and auxiliaries possess at least structural valence and, thus, are regentia in respect to the hierarchically "lower" situated so called "full verbs" (cf. Eroms 2000). In this case, non-finite sentences have to be considered as elliptical ones and treated accordingly to this status. This very complicated and complex theoretical problem of the dependence grammar is the subject of this paper containing an analysis of examples from various languages and different stages of their development, i.e. from synchronic, diachronic and typological perspectives.

Keywords: dependence, valency, sentence, proposition, finiteness, non-finiteness, verb-centric syntax.

\section{Zum Status der Finitheit im Satz}

Die Standardwerke zur deutschen Grammatik behandeln das Merkmal der Finitheit fast ausnahmslos als ein unveräußerliches Kriterium für die Satzkonstituierung. Als Satz gilt demgemäß ein Syntagma, dessen Prädikat eine synthetische Verbalform enthält, vgl.

(1) Um die Ecke wuchsen Linden.

(2) Die Versammlung hat um neun Uhr vormittags begonnen.

(3) Die angereisten Touristen mussten eine halbe Stunde auf den Shuttlebus warten.

(4) Würden Sie mir darüber genauer erzählen?

(5) Komm doch näher!

Dagegen werden inhaltlich satzwertige Propositionen ohne Finitum nicht als Sätze, sondern als Minimaläußerungen (vgl. Darski 2010: 95-98) bzw. kommunikative Minimaleinheiten eingeordnet (vgl. u.a. Zifonun 1987: 34-64, Zifonun et al. 1997, Bd. 1), da sie zwar - wie die echten Satzsyntagmen - komplexe prädikative Sachverhalte kodieren und eine selbständige illokutive Kraft haben, andererseits aber - im Gegensatz zu den „echten“ Sätzen - keine Finitheit besitzen, vgl.

(6) Die Bundeskanzlerin gestern Abend in Warschau eingetroffen.

(7) Herzlich willkommen an unserer Hochschule!

(8) Auf zum Kampf!

(9) Alle Türen und Fenster umgehend schließen, bitte!

(10) Ich - und weinen?

Die Bestimmung des Status afiniter Konstruktionen kann ohne eine Präzisierung des theoretischen Rahmens, in dem sie erfolgt, wohl kaum erfolgreich sein. Beschränken wir uns auf die Syntax der deutschen Sprache, ist das Konzept einer hierarchisch übergeordneten Finitheit durchaus akzeptabel. Im Deutschen spielt sie nämlich eine derart zentrale Rolle, dass die Interpretation der meisten satzartigen Gebilde ohne finit kodierte Prädikationen als grundsätzlich elliptische Strukturen, die auf Prädikationen mit einem finiten Verb zurückgeführt werden können, gerechtfertigt ist. In (6) ist die Kopula ist problemlos nachzutragen; in (7) „fehlt" ibr seid bzw. Sie sind; in (8) brich bzw. brecht/ brechen Sie. Doch schon in (9) und (10) entstehen Probleme bei einer „Ergänzungsprobe“. Ihr sollt / Sie sollen in (9) vor dem vermeintlich elliptischen Satz sind zwar legitim, aber es ist zu zweifeln, ob die Illokution in diesem Fall absolut unverändert bleibt: Offenkundig wird z.B. die Direktheit der Aufforderung und ihre „Abruptivität" und Eindringlichkeit durch die Verwendung des Modalverbs sollen abgeschwächt. Der Satz (10) lässt sich seinerseits überhaupt kaum als Ellipse einordnen. Aber 
in vielen anderen Sprachen ist dieses Problem noch viel stärker ausgeprägt und auf dem Weg einer Ellipse-Ergänzung zum angeblich „dahinter stehenden“ „Vollsatz“ eher unlösbar. Sehr viele Sprachen lassen z.B. keine 'sein'-Kopula in der temporalen Gegenwartsform zu, vgl. russ.:

(11) Gogol - velikij russkij pisatel'.

Gogol großer russischer Schriftsteller

„Gogol ist ein großer russischer Schriftsteller.“

Nachgetragen kann hier das kopulative 'sein'-Verb nur rein abstrakt, aber die Satzoberfläche schließt seine Verwendung aus, da sie den Satz ungrammatisch machen würde. Dies ist nur ein, wenngleich sehr anschauliches, Beispiel dafür, dass bei einer Verallgemeinerung des Finitheitskriteriums und seiner Verabsolutierung und Universalisierung sogar zentrale Minimalsätze (Kopula-Prädikativ-Konstruktionen), welche seit der Antike zu Recht als prototypische Sätze gelten, in ihrer denkbar neutralen Form eben nicht als Sätze, sondern als Minimaläußerungen behandelt werden sollten. Die Kuriosität dieses Konzepts verschärft sich mehrfach, wenn man bedenkt, dass die Vergangenheits- und Zukunftsformen dieser Aussagen im Gegensatz zu den Gegenwartsformen einer Kopula unbedingt bedürfen und demgemäß dem Finitheitskriterium genügen, also „echte“ Sätze sind, vgl.

(12) Gogol' byl velikim russkim pisatelem.

Gogol war groß-Instr. russisch-Instr. Schriftsteller-Instr.

"Gogol war ein großer russischer Schriftsteller."

Afinitheit ist in den Sprachen wie Russisch dermaßen stark vertreten, dass ganze recht umfangreiche Texte ohne ein einziges finites Verb durchaus möglich sind. Folgt man dem Kriterium der Finitheit als einem universellen unveräußerlichen satzkonstituierenden Merkmal, sollen Texte wie der unten stehende (vgl. Kotin 2007: 195) als komplexe Gebilde behandelt werden, die keinen einzigen Satz enthalten und lediglich aus Minimaläußerungen ohne Satzstatus bestehen:

(13) Иван Андреевич, пожалуй, мучший окулист в нашем городе. Ему просто нет равных. У него на приёме всегда много пациентов. Немало сложных случаев. И несмотря на это его диагноз всегда точен, а назначаемое мечение эффективно. Колмеги доктора убеждены, что один день рядом с Мастером - всё равно, что месяц стажировки даже в столичной клинике. Несмотря на свой талант и непререкаемый авторитет Иван Андреевич на удивление скромный чемовек. А ведь сколько житемей нашего города благодарны ему за помощь! Побольше бы таких врачей, как Иван Андреевич Петров!

„Ivan Andrejevič [ist] wahrscheinlich der beste Augenarzt unserer Stadt. Es [kann] wohl keiner mit ihm verglichen [werden]. In seiner Sprechstunde [sind] immer viele Patienten. Nicht selten [gibt es] schwierige Fälle. Trotzdem [ist] sein Befund immer präzise und die verschriebenen Arzneimittel [sind] effizient. Die Kollegen des Doktors [sind] überzeugt, dass ein einziger Tag mit dem Meister dasselbe, was ein ganzer Monat Praktikum, selbst in einer Hauptstadt-Klinik [bringt]. Trotz seines Talents und seiner unumstrittenen Autorität [ist] Ivan Andrejevič ein sehr bescheidener Mensch. Und wie viele Menschen [sind] ihm dabei für seine Hilfe dankbar! Wenn [es] nur mehr solche Ärzte [gäbe], wie Ivan Andrejevič Petrov!“

Wenn wir nun die Syntax mit N. Chomsky als „the study of the principles and processes by which sentences are constructed in particular languages" (zitiert nach Jacobs et al. 
1993: 1) verstehen, müssten die Konstruktionen dieses Typs streng genommen von der Syntaxforschung ausgeschlossen werden. Dabei stellt Chomsky zu Recht fest (zitiert nach Jacobs 1993: 1):

"The ultimate outcome of these investigations should be a theory of linguistic structure in which the descriptive devices utilized in particular grammars are presented and studied abstractly, with no specific reference to particular languages".

Offenkundig kann Finitheit unter dieser Maßgabe nicht als universelles Satzkriterium akzeptiert werden. Hierbei handelt es sich natürlich lediglich um das formale Merkmal - Vorhandensein eines finiten Verbs als unveräußerliches Satzkriterium und nicht etwa um Aussparung der Finitheit gerade bei - temporal merkmallosen - Sätzen mit Gegenwartsbezug. Im anderen Fall müsste man gerade das Feblen overter Finitheit als merkmalloses Satzkriterium mit Gegenwartsbezug annehmen, was weder empirisch noch theoretisch haltbar wäre.

\section{Dependenz und Valenz in der Syntaxforschung - zum Status des Verbs im Satzgebilde}

L. Tesnière 1959/1976 hat die wichtigsten Postulate einer dependentiellen Syntaxtheorie formuliert. Die erste Metapher (Valenz) kommt aus der Chemie: Der Satz wird mit einem chemischen Stoff bzw. einer chemischen Verbindung verglichen, welche durch Anschluss von Atomen an andere, valenztragende Atome chemischer Elemente gebildet werden. Die zweite Metapher stammt aus dem Theater. Das Wichtigste an einem Theaterstück ist die Handlung, welcher im Satz das Verb entspricht. Sie dominiert über die Teilnehmer der Handlung (Akteure), die in etwa den Objekten (Aktanten) entsprechen. Darüber hinaus gibt es Kulissen und andere „Umstände“, die die Handlung begleiten - im Satz sind es die Cirkonstanten, zu denen vor allem Adverbiale verschiedener Art gehören (vgl. u.v.a. Ágel 2000: 74; 113; Eroms 2001: 154; 158). Freilich sind derartige Analogien, da sie ausgesprochen metaphorisch sind, lediglich als terminologische Stützen bzw. Merkhilfen zu behandeln. So hätte z.B. eine volle Analogie mit der chemischen Valenz nie zu einer streng verbozentrischen Konzeption führen können, gibt es ja in der Chemie auch sog. kovalente Relationen, welche durch „gleichberechtigte" Wertigkeit beider Elemente gekennzeichnet sind etc. (vgl. Demjankov 1980: 339). Diese Bemerkung Demjankovs entkräftet natürlich keinesfalls die Angemessenheit der verwendeten Termini, aber sie lässt u.a. - parallel zur Chemie - eventuelle kovalente Relationen im Satzverbund als Option bedenken. In seinem Werk behandelt Tesnière zwar ausschließlich das Verb als Satznukleus (Regens) und sämtliche davon abhängigen Satzglieder als Aktanten bzw. Cirkonstanten, aber seine Theorie schließt keinesfalls aus, dass auch andere Wortarten (z.B. Substantiv oder Person-Numerus-Flexem) im Prinzip als Satznuklei auftreten können, was übrigens der Geehrte zu recht betont (vgl. Engel 2006: 309). Diese Einschränkung ist äußerst wichtig, da sie eine angemessene Konstellation von Universalgrammatik und idioethnisch konzipierten syntaktischen Modellen vorprogrammiert. Die „regierende“ Funktion des Verbs wird - im Unterschied zu dem Universalitätsanspruch der generativistischen Phrasen-Zweiteilung - nicht verabsolutiert, sondern lediglich für konkrete Sprachen in einem 
konkreten Stadium ihrer Entwicklung postuliert, sie trägt also vielmehr „idiomatischen“ als „universell-systematischen“ Charakter (vgl. Engel 2006: 309):

„Zwar hat Tesnière eine dependentielle Verbgrammatik geschrieben, aber sein Dependenzprinzip ist durchaus für andere Sehweisen offen. Es steht nirgends geschrieben, dass in Dependenzgrammatiken das Verb im Zentrum stehen muss."

Es fällt trotzdem auf, dass Tesnière im Unterschied zu Chomsky in seinem Modell gänzlich auf eine Phrasen-Binarität (NP vs. VP) verzichtet - zu Gunsten einer Regens-Dependens-Relation zwischen einer hierarchisch „höheren“ Entität und einer bzw. mehreren davon abhängigen Entitäten.

Dieses Charakteristikum der Dependenzsyntax gestattet eine angemessene einzelsprachlich begründete Phrasen- bzw. Satzmodellierung, die im Spannungsfeld zwischen begrifflich konzipierter Prädikatenlogik und empirischen Evidenzen natürlicher Sprachen liegt, vgl. die folgende Feststellung von H.-W. Eroms (1981: 27):

„Das Valenzkonzept sollte [...] zunächst einzelsprachlich begründet und die möglichen Generalisierungen sollten von daher aufgesucht werden.“

Bezüglich des in diesem Beitrag behandelten Problems ist diese Feststellung insofern wichtig, als sie eine methodologisch haltbare Lösung des „Finitheits-Dilemmas“ zu bieten vermag. Unter dieser Maßgabe wäre die Vorstellung berechtigt, nach der ein Syntagma ohne finites Verb in der Sprache X (wo die Finitheit generell zumindest strukturell einen Sonderstatus besitzt) als „Nicht-Satz“ (sondern z.B. lediglich als eine Minimaläußerung) zu behandeln wäre, wohingegen sie in der Sprache Y mit einem anderen systemischen Status der Finitheit durchaus als vollwertiger Satz gelten könnte. Eine andere methodologische Frage ist natürlich, ob man derart generelle Definitionen wie die Satzdefinition um den Preis des Verzichts auf universalgrammatische Generalisierungen erkaufen darf.

Offensichtlich sind Sätze und schlechthin Syntagmen grundsätzlich hierarchisch organisiert. In den Syntaxtheorien verschiedener Prägungen wird darüber diskutiert, welcher Art diese Hierarchien sind, ob sie z.B. primär formal oder primär semantisch beschaffen sind, wie diverse hierarchische Relationen (Koordinationen/Subordinationen, eng bzw. weit verstandene Rektion, Bindung, Anschluss etc.) im Rahmen einer linearen Syntagmenstruktur interagieren, welche davon „ursprünglich“ und welche „abgeleitet“ sind etc. Seit dem letzten Viertel des 20. Jh. wird immer häufiger nicht nur ,allgemein mental“, sondern geradezu neurobiologisch und -physiologisch argumentiert, um eine universalgrammatisch basierte Syntax zu begründen. So wird u.a. bei W.P. Lehmann (1978: 9) das „Linke-Hemisphäre-Argument“ benutzt, um das verbozentrische Konzept neurobiologisch zu untermauern:

"The studies have indicated that while the right, nondominant hemisphere can manage utterances of nouns, especially concret nouns, only the left, dominant hemisphere can manage the utterance of verbs. Since human language is intimately connected with specially developed sections of the left hemisphere which have the unique capability of controlling verbs as well as the information conveyed through nouns, we have nonlinguistic evidence to support the linguistically based conclusion that the verb is the most characteristic segment of human language. Linguistic typology then must concern itself centrally with the verb and its constructions". 
Eine nähere Betrachtung dieser überraschend generalisierten Schlussfolgerung zeigt eine Reihe auffälliger Probleme. Das „Verb“ wird generell in der linken Gehirn-Hemisphäre platziert - ohne Erläuterung, ob es sich dabei um seine allgemeinen strukturell-transformationellen Eigenschaften oder auch um konkrete lexikalische Bedeutungen der jeweiligen verbalen Entitäten handelt. Es muss daher angenommen werden, dass die linke Hemisphäre für beides verantwortlich ist. Wir bekommen damit ein strukturell-semantisches Modul. Es steht dem strikten (tiefen)strukturellen Modul des frühen Chomsky gegenüber und ist mit dem semantisch stärker geprägten Modul des späteren Chomsky und seiner Schüler nur zum Teil affin, da es die Nominalphrase aus der linken Hemisphäre in starkem Maße in die rechte, nicht ,sprachdominierte“, verdrängt. Für das Government-Binding-Modell ist diese Trennung unvertretbar, vor allem wegen der darin zentralen „Sonderrolle“ des Subjekts in der Subkategorisierungsstruktur des Satzes (vgl. u.v.a. Abraham 2005: 21). Dagegen ist es mit dem Valenzmodell Tesnièrescher Prägung und mit sonstigen strikt verbozentrischen Dependenzmodellen durchaus kompatibel, obwohl insgesamt dennoch der Eindruck entsteht, dass die Grenze zwischen „sprachdominiertem“ Verb und „nicht-sprachdominiertem Nomen" zu scharf gezogen ist: Die Regentia dominieren nicht nur die Dependentia, sie sind im Grunde genommen allein für die „Sprache“ verantwortlich. ${ }^{2}$

Doch auch bezüglich des Verbs selbst gibt es hierbei keine befriedigende Lösung. Was gilt als „Verb“ in Satzsyntagmen mit mehrteiligen Prädikaten bzw. welches „Verb“ muss als Valenzträger aus semantischer und aus struktureller Sicht eingeordnet werden? Sind Modal-, Modalitäts- bzw. Auxiliarverben Träger einer zumindest strukturellen Valenz, wie u.a. H. Wegener (1990: 157-159) oder H.-W. Eroms (2000: 140) annehmen, oder sind sie grundsätzlich nicht valenzfähig und somit völlig in das Valenzschema des jeweiligen „Vollverbs“ integriert, da doppelte Abhängigkeiten generell ausgeschlossen seien? (vgl. Engel 1988: 630).

Betrachten wir den Satz Wolfgang kann schwimmen. Bei der Bestimmung von Dependenzrelationen, die diesem Satzsyntagma zu Grunde liegen, gibt es zwei Möglichkeiten, nämlich (i) Wolfgang [kann schwimmen] und (ii) Wolfgang kann schwimmen, also X - V vs. X - V - Y. Im ersten Fall wird dem Modalverb eine selbständige (satzbildende) Valenz abgesprochen, und das Gesamtsyntagma wird als Projektion des (Voll)verbs schwimmen behandelt. Das Prädikat, welches aus dem finiten Modalverb und dem Infinitiv des Vollverbs besteht, wird demgemäß als ein zusammengesetztes (verbales) Prädikat behandelt mit dem Vollverb als Kopf. Diese Ansicht kann als „traditionell“ bezeichnet werden, da die Tradition der Einordnung von Modalverben als unselbständige Prädikatsteile in der Syntaxforschung seit langer Zeit existiert und auf jeden Fall älter als Dependenzgrammatik ist. In die Valenztheorie wurde diese Ansicht u.a. von U. Engel (1988: 463-464) ,übertragen“, d.h., sie wurde von Engel valenztheoretisch begründet. Die Tatsache, dass das Subjekt mit dem Modalverb kongruiert, betrachtet

2 Die angeführte Argumentation betrifft freilich eine Etappe in der Entwicklung des universalgrammatischen Denkens, die heutzutage bereits Geschichte ist, da die bildgebenden Verfahren inzwischen viel präziser geworden sind (vgl. u.a. die genauen Ausführungen diesbezüglich bei Černigovskaja 2013: 65-76; 221-315; 319-325). Doch scheint sie hier wichtig wegen einer allgemeinen „Symptomatik“, welche die universaltypologische bzw. universalgrammatische Denkweise charakterisiert, wenn sie „Argumentationsstützen“ aus dem neurobiologischen Bereich sucht. Diese Stützen gibt es auch tatsächlich, allerdings sind sie weder linear noch eindeutig und endgültig feststellbar. 
Engel „als eine Oberflächenerscheinung, ein sekundäres Phänomen“ (ibid.). Das Subjekt hängt in derartigen Syntagmen nach Engel lediglich vom Infinitiv des Vollverbs ab, da gerade der Infinitiv darüber entscheidet, ob ein Subjekt überhaupt selegiert wird. So erfordert der subjektlose Satz Dich kann frieren. kein Subjekt, da das Vollverb frieren in Dich friert. kein Subjekt erfordert (zur Auseinandersetzung mit dieser Ansicht vgl. meine Ausführungen in Kotin 2007: 143-145). Die Gegenansicht vertreten u.a. Welke (1988: 149-151) und Eroms (2000: 141-145), die davon ausgehen, dass der Infinitiv eine Ergänzung des Modalverbs darstellt. Diese Auffassung beruht auf der bekannten Gliederung der Rektion von Modal- und Modalitätsverben in Kasus- und Statusrektion, die von G. Bech (1955-1957) unternommen und von J.O. Askedal (vgl. Ágel et al. 2006: 892-893) an die Valenzgrammatik adaptiert wurde.

\section{Zur Stellung der Finitheit und Afinitheit in der Dependenz- bzw. Valenzgrammatik}

Bei einfachen (einteiligen) Prädikaten deckt sich die Valenz des Verbs auf Systemebene in aller Regel weitestgehend mit der Valenz des Prädikats auf der Ebene der Parole. Bei zusammengesetzten (mehrteiligen) Prädikaten, aber auch bei analytischen Tempus-, Modus- oder Genusformen (grammatischen Verbalperiphrasen) hängt die Bestimmung der Valenzeigenschaften der Einzelteile von mehreren Faktoren ab, deren Bestimmung jeweils theoriekonform sein muss, damit keine internen Widersprüche bei ihrer Deskription entstehen. Im vorhergehenden Unterkapitel wurde nämlich gezeigt, dass den Modal- und Auxiliarverben eigene Valenz sowohl zu- als auch abgesprochen werden kann, je nachdem, wie ernst deren strukturell-syntaktische Wertigkeit (ggf. auch ohne eine angemessene „semantische Füllung“) genommen wird. Jede jeweils alternative Vorgehensweise hat ihre Vor- und Nachteile. Bei „unifizierendem“ Herangehen wird das Prädikat weitgehend dem Vollverb gleichgestellt, was eine einheitliche Systematik der Paradigmatik und Syntagmatik sichert, wohingegen das "kompositionelle“ Herangehen eine kompliziertere Beziehung zwischen Form und Funktion komplexer Prädikatszeichen voraussetzt.

Für Syntagmen ohne Verbum finitum kann unter dieser Maßgabe generell entweder der Ellipse-Status oder aber der Status einer autonomen Proposition postuliert werden. Ellipsen sind per definitionem nichts Anderes als unvollständige Sätze, welche durch relativ einfache Prozeduren zu Vollphrasen ergänzt werden können, während autonome afinite Propositionen als eigenständige Sätze einzuordnen sind, die sich von „finiten“ Sätzen durch gewisse Merkmale unterscheiden, die logischerweise von der Theorie zu eruieren wären.

Betrachten wir den folgenden gotischen Beleg aus dem Matthäus-Evangelium, Kapitel 8, Vers 1:

dalap pan atgaggandin imma af fairgunja laistidedun afar imma iumjons managos.

hinunter dann steigendem ihm von Berg folgten nach ihm Mengen viele

"Als er dann vom Berg hinunter stieg, folgten ihm viele Menschen."

Der gotische Satz kopiert weitgehend die griechische Vorlage. Sein erster Teil ist eine absolute 
syntaktische Konstruktion, der so genannte Dativus absolutus, welcher ins moderne Deutsch immer als ein Temporalsatz übersetzt wird, der durch die Subjunktion als eingeleitet wird. Im Griechischen entspricht dieser Form der so genannte Genitivus absolutus, im Lateinischen der Ablativus absolutus und im Altkirchenslawischen wird, ähnlich zum Gotischen und z.T. zum Althochdeutschen, der absolute Dativ verwendet. Die Gesamtkonstruktion besteht in aller Regel aus einem Substantiv oder Pronomen in Verbindung mit dem Partizip I oder II des verbalen Prädikats, jeweils im Genitiv (Griechisch), Ablativ (Latein) oder Dativ (Altgermanisch, Altkirchenslawisch), vgl. ahd. (Tatian 45, 2 - Joh. 2, 3):

thô ziganganemo themo uúine quad thes heilantes muoter zi imo: sie ni habent uúin. da vergangenem dem Wein sagte des Heilands Mutter zu ihm sie nicht haben Wein

,als es an Wein mangelte [wörtl. ,dann zu Ende gegangenem dem Wein'], sprach die Mutter des Heilands zu Ihm: sie haben keinen Wein (mehr):

oder got. Mt. 8, 16:

at andanahtja pan waurpanamma atberun du imma daimonarjans managans [...]

(auf $)^{3}$ Abend dann gewordenem brachten zu ihm Besessene viele

,als dann Abend kam, brachte man zu ihm viele Besessene [... $]^{c}$

Offenkundig liegen in allen Belegen mit absoluten Tempuskonstruktionen jeweils zwei autonome Propositionen vor, von denen die jeweils erste afinit ist. Es gibt keine Gründe, den Status der absoluten Konstruktion und des als-Gliedsatzes mit finiter Verbform unterschiedlich zu bewerten. Die Valenzeigenschaften der Partizipien werden dabei auch beim Fehlen des finiten Teils des Prädikats voll realisiert. Eine ähnliche Situation liegt im Falle afiniter Sätze vor, vgl. die folgende Stelle aus dem Gedicht von Fr. Schiller „Ideale“:

Erloschen sind die heit'ren Sonnen, / die meiner Jugend Pfad erhellt, / Die Ideale sind zerronnen, / die einst das trunk'ne Herz geschwellt.

Im jeweils ersten Satz beider Zeilen ist das finite Verb sein vorhanden, sodass die Tempusform des Perfekts in ihrer vollen Gestalt auftritt. Das Auxiliarverb ist Träger struktureller Valenz, während die semantische Wertigkeit vom Partizip II kodiert wird. Im jeweils zweiten Satz lässt sich das Finitum problemlos ergänzen, wobei sogar seine Präteritalform deutlich prognostiziert werden kann, wodurch das Prädikat des jeweiligen Relativsatzes ohne Zweifel im Plusquamperfekt zu lesen ist: , die meiner Jugend Pfad erhellt hatten bzw. , die einst das trunk'ne Herz geschwellt hatten. Es handelt sich somit um eine Referenzzeit (Vorzeitigkeit in der Vergangenheitsstufe) gemäß dem bekannten Reichenbach-Schema (vgl. Reichenbach 1947/21965), die auch ohne overtes Auxiliar rekonstruiert werden kann. Ebenso kann die Perfektform mit haben (in Verbindung mit dem overten Präsens) im folgenden Beleg aus Goethes „Zueignung“ zum „Faust“ rekonstruiert werden:

Ihr naht euch wieder, schwankende Gestalten, / Die früh sich einst dem trüben Blick gezeigt [habt]

In seinem Vortrag auf der GGSG-Tagung in Siegen im September 2012 hat J. Riecke am Korpus

3 Die Präposition at in Verbindung mit dem Dativus absolutus hat eine spezifische temporale Funktion und darf daher nicht wörtlich übersetzt werden (vgl. Streitberg 1965/2: 13). 
der Kriegstagebücher F. Kellners gezeigt, dass die in der historischen Syntax für aufgegeben erklärte Afinitheit und damit vergleichbare Phänomene (darunter die Infinitiv-Weglasssung) auch in einem Text aus der Mitte des 20. Jh. durchaus anzutreffen sind, und zwar geradezu sehr häufig, vgl. (Belege nach dem Handout von Riecke 2012):

3.12.1943

Was vergangen, kehrt in der gleichen Gestalt nie wieder.

16.4.1940

Wenn das deutsche Volk einmal in seinem ganzen Umfange die ihm aufgetischten

Märchen u. Indianergeschichten als das erkannt, was sie sind - dann ist es allerdings

zu spät.

Keiner der Belege lässt Zweifel bezüglich der Ergänzung von finiten Auxiliaren, einschließlich ihrer Tempusformen, bzw. des ausgelassenen Infinitivs zu. Es sind: ist im ersten, hat im zweiten Beleg.

Der grundsätzliche Unterschied zwischen den letztgenannten Sätzen und früher behandelten afiniten Propositionen besteht jedoch darin, dass afinite Sätze in absoluten Konstruktionen keine Ellipsen im üblichen Sinn dieses Begriffs darstellen, d.h. nicht einfach durch Zusatz eines Finitums ergänzt werden können. Sie können lediglich komplett umgestaltet werden, in unserem Fall mithilfe eines eingeleiteten Temporalsatzes. Nichtsdestoweniger können beide Typen afiniter Prädikationen durch Vollentfaltung der Valenzeigenschaften des infiniten Verbteils (Partizips) als komplette Satzsyntagmen eingeordnet werden. Was nun Sprachen betrifft, in denen die Kopula unter bestimmten Bedingungen (z.B. in Existenzsätzen in der Gegenwartsform) ausgelassen werden muss (vgl. Unterkapitel 1, Belege (11) und (13)), können sie als „koverte Ellipsen" behandelt werden in dem Sinn, dass hier die Nullhypothese die Auslassung der Kopula als Indikator ihres inhärenten Vorhandenseins in der Tiefenstruktur einer denkbar neutralen Prädikation (hier der Gegenwartsform) voraussetzt.

\section{Fazit}

Wird das Phänomen der Afinitheit im Rahmen der Dependenzgrammatik bzw. Valenztheorie aus universalgrammatischer Sicht behandelt, muss festgestellt werden, dass die Finitheit wohl kaum als konstituierendes Satzkriterium gelten kann. Dies ist selbst bei Sprachen wie Deutsch mit stark indizierter overter Finitheit problematisch, da die Valenzeigenschaften nicht finiter Prädikatsteile darin in aller Regel voll ausgeprägt sind. Umso stärker sind in- und afinite Syntagmen in den Sprachen mit indizierter koverter Finitheit Vollsätze.

\section{Literatur}

Abraham, Werner (2005): Deutsche Syntax im Sprachenvergleich. Grundlegung einer typologischen Syntax des Deutschen. Tübingen: Stauffenburg.

Ágel, Vilmos (2000): Valenztheorie. Tübingen: Narr. 
Ágel, Vilmos / Eichinger, Ludwig M. / Eroms, Hans-Werner / Hellwig, Peter / Heringer, Hans-Jürgen / Lobin, Henning (Hg.) (2006): Dependenz und Valenz. Ein internationales Handbuch zeitgenössischer Forschung Halbbd. 2. Berlin / New York: de Gruyter.

Bech, Gunnar (1955-1957): Studien über das deutsche Verbum infinitum. Bde. 1-2. Kopenhagen: Munksgaard.

Černigovskaja, Tatjana V. (2013): Češirskaja ulybka kota Šrëdingera. Jazyk i soznanije. Moskva: Jazyki slavjanskoj kul'tury.

Darski, Józef P. (2010): Deutsche Grammatik. Ein völlig neuer Ansatz. Frankfurt/M.: Peter Lang.

Demjankov, Valerij Z. (1980): Predikaty i konzepcija semantičeskoj interpretacii. In: Izvestija Akademii Nauk SSSR, Serija literatury i jazyka 4 (1980), 336-346.

Engel, Ulrich (1988): Deutsche Grammatik. 2. Aufl. Heidelberg: Groos.

Engel, Ulrich (2006): Gaudium in scientia linguarum. Ausgewäblte Schriften. hrsg. von Alina Jurasz et al. Wrocław / Dresden: ATUT / Neisse Verlag.

Eroms, Hans-Werner (1981): Valenz, Kasus und Präpositionen. Untersuchungen zur Syntax und Semantik präpositionaler Konstruktionen in der deutschen Gegenwartssprache. Heidelberg: Winter.

Eroms, Hans-Werner (2000): Syntax der deutschen Sprache, Berlin / New York: de Gruyter.

Jacobs, Joachim / Stechow von, Arnim / Sternefeld, Wolfgang / Vennemann, Theo (Hg.) (1993): Syntax. Ein internationales Handbuch zeitgenössischer Forschung. Halbbd. 1, 2. Berlin / New York: de Gruyter.

Kotin, Michail L. (2007): Die Sprache in statu movendi. Sprachentwicklung zwischen Kontinuität und Wandel. Zweiter Band: Kategorie - Prädikation - Diskurs. Heidelberg: Winter.

Kotin, Michail L. (2014): Makrolinguistik. Möglichkeiten und Grenzen der Theoriebildung bei der Textbzw. Diskurslinguistik. In: Bassola, Péter / Drewnowska-Vargáné, Ewa / Kispál, Támas / Németh, János / Scheibl, György (Hg.): Zugänge zum Text. Frankfurt/M.: Peter Lang Edition, 273-293.

Lehmann, Winfred P. (ed.) (1978): Syntactic Typology. Studies in the Phenomenology of Language. Sussex: The Harvester Press.

Reichenbach, Hans (1947 / $\left.{ }^{2} 1965\right)$ : Elements of Symbolic Logic. New York: Mc Millan.

Riecke, Jörg (2012): Beobachtungen zur Sprache der Kriegstagebücher Friedrich Kellners (1939-1945). Abstract und Handout zum Vortrag auf der Jahrestagung der Gesellschaft für Germanistische Sprachgeschichte (GGSG) in Siegen.

Streitberg, Wilhelm (Hg.) (1965): Die gotische Bibel. Erster Teil: Der gotische Text und seine griechische Vortlage. 5., durchges. Aufl. Zweiter Teil: Gotisch-griechisch-deutsches Wörterbuch. 4., unveränderte Aufl. Heidelberg: Winter.

Tesnière, Lucien (1959 / $\left.{ }^{2} 1976\right)$ : Eléments de syntaxe structurale. 2. Aufl. Paris: Klincksieck.

Wegener, Heide (1990): Komplemente in der Dependenzgrammatik und in der Rektions- und Bindungstheorie: Die Verwendung der Kasus im Deutschen. In: Zeitschrift für germanistische Linguistik 18 (1990), 150-184.

Welke, Klaus M. (1988): Einführung in die Valenz- und Kasustheorie. Leipzig: VEB Bibliographisches Institut.

Zifonun, Gisela (1987): Kommunikative Einheiten in der Grammatik. Tübingen: Narr.

Zifonun, Gisela et al. (Hg.) (1997): Grammatik der deutschen Sprache. 3 Bde. Berlin: de Gruyter. 